\title{
Electrical interfacing of neurotransmitter receptor and field effect transistor
}

\author{
I. Peitz and P. Fromherz ${ }^{\mathrm{a}}$ \\ Department of Membrane and Neurophysics, Max Planck Institute for Biochemistry, D 82152 Martinsried/Munich, Germany
}

Received: 5 November 2008

Published online: 10 June 2009 - (C) EDP Sciences / Società Italiana di Fisica / Springer-Verlag 2009

\begin{abstract}
The interfacing of a ligand-gated ion channel to a transistor is studied. It relies on the transduction of ion current to a voltage in a cell-transistor junction. For the first time, a genetically modified cell is used without external driving voltage as applied by a patch-pipette. Using a core-coat conductor model, we show that an autonomous dynamics gives rise to a signal if a driving voltage is provided by potassium channels, and if current compensation is avoided by an inhomogeneous activation of channels. In a proof-of-principle experiment, we transfect HEK293 cells with the serotonin receptor 5-HT3A and the potassium channel Kv1.3. The interfacing is characterized under voltage-clamp with a negative transistor signal for activated 5-HT3A and a positive signal for activated Kv1.3. Without patch-pipette, a biphasic transient is induced by serotonin. The positive wave is assigned to 5-HT3A receptors in the free membrane that drive a potassium outward current through the adherent membrane. The negative wave is attributed to 5-HT3A receptors in the adherent membrane that are activated with a delay due to serotonin diffusion. The implementation of a receptor-cell-transistor device is a fundamental step in the development of biosensors that combine high specificity and universal microelectronic readout.
\end{abstract}

PACS. 87.85.dh Cells on a chip - 85.30.Tv Field effect devices - 87.16.Vy Ion channels

\section{Introduction}

The interfacing of nerve cells to semiconductor chips relies on a coupling of their electrical excitation to transistors [1]. A more sophisticated level of interfacing would be attained, if the chemical signals of neuronal excitation - neurotransmitters - were electronically detected, too. We could envisage not only components for future neurocomputing systems, but also sensors for drug development (high-throughput screening) and environmental analysis (neurotoxin detection). In the present paper we describe how the physical problem of signal transduction is solved without control of the intracellular voltage by a patch-pipette in a receptor-cell-transistor device for an ionotropic serotonin receptor.

Figure 1 illustrates the principle for transistor recording of receptor-activated ion channels in a cell membrane. When the channels are opened by a specific ligand, ionic current enters the cell. The current through the adherent membrane flows along the narrow cleft between cell and transistor. It gives rise to an extracellular voltage $V_{J}$ in the cell-transistor junction with respect to the bath. That voltage represents a change of the gate voltage of the field effect transistor and modulates its source drain current.

\footnotetext{
a e-mail: fromherz@biochem.mpg.de
}

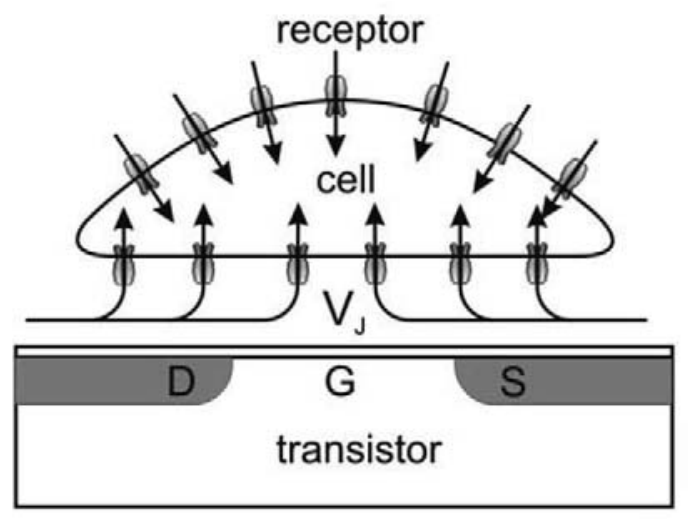

Fig. 1. Interfacing of ligand-gated ion channels in a receptorcell-transistor device (not to scale). A cell (diameter around $20 \mu \mathrm{m}$ ) is separated from an electrolyte-oxide-semiconductor field effect transistor (EOSFET) by a narrow cleft (width around $50 \mathrm{~nm}$ ). Ion current enters the cell through channels that are activated by a specific ligand. The current through the attached membrane gives rise to an extracellular voltage $V_{J}$ on the open gate $\mathrm{G}$ of a transistor with source $\mathrm{S}$ and drain $\mathrm{D}$.

In the past, transistor recording of voltage-gated ion channels has been implemented at constant intracellular voltage defined through a patch-pipette. The 
outward current through the potassium channels hSlo and Kv1.3 [2,3] was detected, as well as the inward current through the sodium channel Nav1.4 [4]. Recently, the inward current through a ligand-gated channel was probed under voltage-clamp for the ionotropic serotonin receptor 5-HT3A [5]. To provide well-defined biophysical conditions, these experiments were performed with human embryonic kidney (HEK293) cells, a common expression system for recombinant ion channels with a low background of intrinsic membrane conductances.

With respect to applications, it is a crucial feature of receptor-cell-transistor recording that it can be scaled up to massive parallel sensor systems using large multitransistor arrays [6]. As a prerequisite of such an approach, however, the patch-pipette must be eliminated. It is the aim of the present study to point out the physical problem of transistor recording without patch-pipette, and to demonstrate in a proof-of-principle experiment that a receptor-cell-transistor sensor can be implemented without patch-pipette. In the experiments we used the serotonin receptor 5-HT3A.

Receptors for serotonin (5-Hydroxy-Tryptamin) play an important role in the peripheral and central nervous system [7]. Clinically, specific blockers are used to inhibit chemotherapy-induced emesis and to treat irritable bowel syndrome [8]. The 5-HT3 receptors are ligand-gated ion channels that consist of five subunits with binding sites for serotonin in their extracellular domain [9]. The 5-HT3A receptor has a particularly simple structure with a single kind of subunit A that forms a homopentameric cation selective channel $[10,11]$.

In the first part of the paper, an electrical model of a receptor-cell-transistor device is considered. It shows that a transistor signal is expected without patch-pipette i) if a driving force for a receptor-activated conductance is provided by a second ion conductance with an opposite direction of current flow, and ii) if a compensation of ion currents in the cell-transistor junction is avoided by a different activation of ion conductances in the adherent and free area of the membrane. The second part of the paper shows how these two problems are overcome for the serotonin receptor 5-HT3A. To solve the first problem, we cotransfected HEK293 cells with the voltage-gated potassium channel Kv1.3 with an inward current $[12,13]$. A solution of the second problem was achieved by the delayed activation of 5-HT3A receptors in the adherent membrane as it is caused by the diffusion of serotonin to the area of cell adhesion [5].

\section{Receptor-cell-transistor sensor}

The electrical nature of a cell-transistor junction can be described as a planar core-coat conductor where a thin sheet of electrolyte is separated from the silicon and from the cytoplasm by insulating films of oxide and lipid, respectively [14]. To derive the crucial relations for a receptor-cell-transistor device, we use a simple onecompartment model $[4,14,15]$. The equivalent circuit of Figure 2 shows that the extracellular voltage $V_{J}$ above

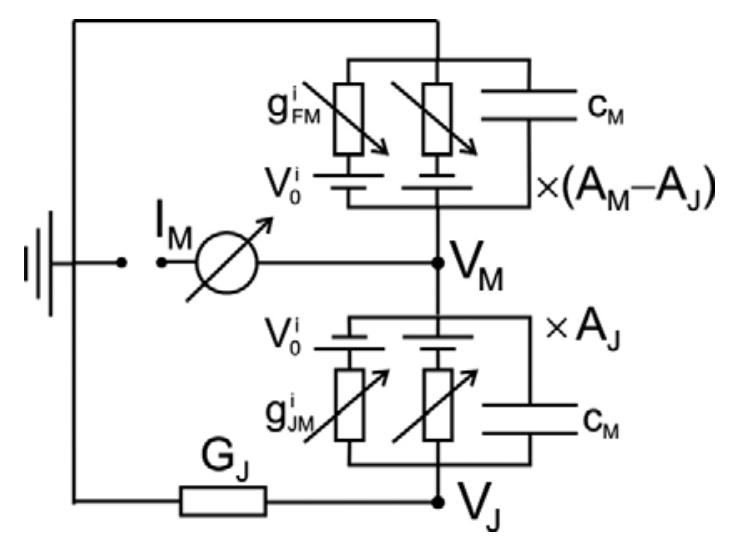

Fig. 2. Equivalent circuit of receptor-cell-transistor device. The upper and lower parts refer to the free and adherent membrane of a cell with areas $A_{M}-A_{J}$ and $A_{J}$, respectively. They consist of an area specific capacitance and two kinds of ion conductances with opposite reversal voltages (receptor-induced conductance and voltage-gated potassium conductance). $G_{J}$ is the conductance of the cell-transistor junction. $V_{M}$ is the intracellular voltage, $V_{J}$ is the extracellular voltage in the celltransistor junction. The horizontal line in the center represents a patch-pipette that is used in test experiments. It records the membrane current $I_{M}$ in the voltage-clamp mode, and the intracellular voltage $V_{M}$ in the current-clamp mode.

the electrolyte-oxide-semiconductor field effect transistor (EOSFET) is determined by the balance of currents through the adherent cell membrane and along the cellchip junction if the capacitive current across the oxide is neglected.

\subsection{Receptor-activated conductance}

\subsubsection{Transistor recording under voltage-clamp}

We consider a cell at a constant intracellular voltage $V_{M}^{V C}$ that is applied by a patch-pipette (voltage-clamp). The total membrane current $I_{M}^{V C}$ is given by equation (1) with the area specific conductances $g_{J M}^{\mathrm{rec}}$ and $g_{F M}^{\mathrm{rec}}$ of a receptoractivated conductance in the adherent and free membrane with the areas $A_{J}$ and $A_{M}-A_{J}$, respectively, at a reversal voltage $V_{0}^{\mathrm{rec}}>V_{M}^{V C}$. From the current balance in the junction, we obtain equation (2) for the extracellular voltage $V_{J}^{V C}$ with a conductance $G_{J}$ of the junction.

$$
\begin{aligned}
& {\left[A_{J} g_{J M}^{\mathrm{rec}}+\left(A_{M}-A_{J}\right) g_{F M}^{\mathrm{rec}}\right]\left(V_{M}^{V C}-V_{0}^{\mathrm{rec}}\right)=I_{M}^{V C},} \\
& A_{J} g_{J M}^{\mathrm{rec}}\left(V_{M}^{V C}-V_{0}^{\mathrm{rec}}\right)=G_{J} V_{J}^{V C} .
\end{aligned}
$$

Equations (1) and (2) hold for small voltages $V_{J}$. For a homogeneous membrane conductance with $g_{F M}^{\mathrm{rec}}=g_{J M}^{\mathrm{rec}}$, we obtain $G_{J} V_{J}^{V C}=I_{M}^{V C} A_{J} / A_{M}$. The extracellular voltage that is recorded with a transistor is proportional to the membrane current that is measured with the patchpipette. 


\subsubsection{Transistor recording without patch-pipette}

In a cell without patch-pipette, a capacitive current balances the current through receptor-activated ion channels. The current balance in the cell is expressed by equation (3) with a capacitance $c_{M}$ per unit area. The extracellular voltage $V_{J}$ is determined by an ionic and a capacitive current according to equation (4).

$$
\begin{aligned}
{\left[A_{J} g_{J M}^{\mathrm{rec}}\right.} & \left.+\left(A_{M}-A_{J}\right) g_{F M}^{\mathrm{rec}}\right]\left(V_{M}-V_{0}^{\mathrm{rec}}\right) \\
& +A_{M} c_{M} \frac{\mathrm{d} V_{M}}{\mathrm{~d} t}=0, \\
A_{J} g_{J M}^{\mathrm{rec}} & \left(V_{M}-V_{0}^{\mathrm{rec}}\right)+A_{J} c_{M} \frac{\mathrm{d} V_{M}}{\mathrm{~d} t}=G_{J} V_{J} .
\end{aligned}
$$

When we eliminate the capacitive current in equation (4) through the adherent membrane with equation (3), we obtain

$$
\frac{A_{M}-A_{J}}{A_{M}} A_{J}\left(g_{J M}^{\mathrm{rec}}-g_{F M}^{\mathrm{rec}}\right)\left(V_{M}-V_{0}^{\mathrm{rec}}\right)=G_{J} V_{J} .
$$

For a homogeneous membrane conductance $g_{J M}^{\mathrm{rec}}=$ $g_{F M}^{\mathrm{rec}}$, there is no extracellular signal because the ionic and capacitive current compensate each other in each area element of the membrane. For an inhomogeneous conductance $g_{J M}^{\mathrm{rec}} \neq g_{F M}^{\mathrm{rec}}$, however, there is no compensation of the currents in the adherent membrane, and a voltage $V_{J} \neq 0$ is induced. Such an inhomogeneity may be caused by an inhomogeneous distribution of ion channels or by an inhomogeneous activation of the channels.

An important feature is not explicitely expressed by equation (5): the extracellular signal $V_{J}(t)$ is a transient. After a sudden activation of the conductance, the intracellular voltage $V_{M}(t)$ rapidly relaxes towards the reversal voltage $V_{0}^{\text {rec }}$ according to equation (3) and the driving voltage of the current is rapidly abolished. If the amplitude of the signal $V_{J}(t)$ is small, such a short transient is difficult to detect with a transistor.

\subsection{Receptor-activated conductance and potassium conductance}

A stationary extracellular voltage $V_{J}$ is induced if the intracellular voltage is kept below the reversal voltage with $V_{M}<V_{0}^{\text {rec }}$. Without patch-pipette, this can be achieved by a voltage-gated potassium channel with a reversal voltage $V_{0}^{K}<V_{0}^{\text {rec }}$.

\subsubsection{Inhomogeneous conductance}

We consider a cell with an inhomogeneous receptoractivated conductance and a homogeneous potassium conductance with $g_{F M}^{\mathrm{K}}=g_{J M}^{\mathrm{K}}=g_{M}^{\mathrm{K}}$. After electrical relaxation, the intracellular voltage results from the balance of ion currents according to equation (6). The extracellular voltage is determined by the ionic current through both conductances of the adherent membrane according to equation (7).

$$
\begin{aligned}
& {\left[A_{J} g_{J M}^{\mathrm{rec}}+\left(A_{M}-A_{J}\right) g_{F M}^{\mathrm{rec}}\right]\left(V_{M}-V_{0}^{\mathrm{rec}}\right)} \\
& \quad+A_{M} g_{M}^{\mathrm{K}}\left(V_{M}-V_{0}^{\mathrm{K}}\right)=0, \\
& A_{J} g_{J M}^{\mathrm{rec}}\left(V_{M}-V_{0}^{\mathrm{rec}}\right)+A_{J} g_{M}^{\mathrm{K}}\left(V_{M}-V_{0}^{\mathrm{K}}\right)=G_{J} V_{J} .
\end{aligned}
$$

In equations (6) and (7), potassium currents replace the capacitive outward currents in equations (3) and (4). When we eliminate the potassium conductance in equation (7) for the adherent membrane with equation (6), we obtain again equation (5): an extracellular signal $V_{J} \neq 0$ appears for $g_{J M}^{\mathrm{rec}} \neq g_{F M}^{\mathrm{rec}}$. Now, however, the intracellular voltage $V_{M}(t)$ is not governed by equation (3) but by equation (6): a stationary intracellular voltage $V_{M} \neq V_{0}^{\text {rec }}$ is established due to potassium channels that are opened by a depolarization induced by receptor-activated channels. That voltage may slowly change due to the desensitization of the receptor-activated conductance and to the inactivation of the potassium channels.

Let us consider two limiting situations: I) A conductance $g_{F M}^{\mathrm{rec}}$ is only present in the free membrane with $g_{J M}^{\mathrm{rec}}=0$. The cell becomes depolarized such that the voltage-gated potassium conductance $g_{M}^{\mathrm{K}}$ is activated in the whole cell. A potassium outward current balances the inward current and keeps the intracellular voltage from the reversal voltage $V_{0}^{\text {rec }}$. A fraction of the potassium current flows through the adherent membrane and gives rise to an extracellular voltage $V_{J}>0$. II) A conductance $g_{J M}^{\mathrm{rec}}$ is only present in the adherent membrane. Again the potassium conductance is activated in the whole cell. The inward current through the adherent membrane is partly balanced by the local potassium outward current. The remainder gives rise to an extracellular voltage $V_{J}<0$.

\subsubsection{Inhomogeneous activation in time}

When a ligand is suddenly applied to the medium around a cultured cell, the receptors in the free membrane are activated almost instantaneously, whereas in the adherent membrane they are activated with a delay due to the diffusion of the agonist to the area of adhesion [5]. In a first stage, a receptor-activated conductance appears in the free membrane with $g_{F M}^{\mathrm{rec}}>g_{J M}^{\mathrm{rec}}$. In a second stage, receptors in the adherent membrane are activated whereas the receptors in the free membrane become desensitized with $g_{F M}^{\mathrm{rec}}<g_{J M}^{\mathrm{rec}}$. The time-dependent signal $V_{J}(t)$ is biphasic with a positive voltage due to a potassium outward current in the adherent membrane and a subsequent negative voltage due to a acceptor-activated current in the adherent membrane.

Let us assume i) that the two stages are well separated in time such that they correspond to the two limiting situations considered above, and ii) that the maxima of the induced conductance are equal in the free and adherent membrane with $g_{J M, \text { max }}^{\mathrm{rec}}=g_{F M, \max }^{\mathrm{rec}}=g_{M, \text { max }}^{\mathrm{rec}}$. From equation (5), we obtain

$$
\mp \frac{A_{M}-A_{J}}{A_{M}} A_{J} g_{M, \max }^{\mathrm{rec}}\left(V_{M}-V_{0}^{\mathrm{rec}}\right)=G_{J} V_{J, \max } .
$$


The negative sign holds for the first stage and the positive sign for the second stage. With $V_{M}<V_{0}^{\text {rec }}$, there is a biphasic signal with a positive and negative wave of equal amplitudes.

\section{Experimental}

\subsection{Materials}

\subsubsection{Chips}

Silicon chips with two linear arrays of low-noise $p$-type EOSFETS were used [16]. They were wire-bonded to a ceramic package. Custom-made laminar-flow chambers (perspex) were attached. The chips were cleaned with an acidic detergent (Ultrax 102 S, KLN, Heppenheim, Germany), rinsed with milli-Q water (Millipore), dried with nitrogen, exposed to hexamethyldisilazane vapor (30 min) and sterilized with UV light $(30 \mathrm{~min})$. The chips were coated with fibronectin (Sigma, $10 \mu \mathrm{g} / \mathrm{ml}$ in PBS, 2 hours, $4{ }^{\circ} \mathrm{C}$ ) to promote cell adhesion and rinsed with PBS (phosphate buffered saline).

\subsubsection{Cells}

We used human embryonic kidney cells HEK293 (DSMZ, Braunschweig, Germany) that stably expressed the voltage-gated $\mathrm{K}^{+}$channel $\mathrm{Kv} 1.3$ [3]. The cells were cultivated at $37^{\circ} \mathrm{C}$ in $5 \% \mathrm{CO}_{2}$ in Dulbecco's modified Eagle's medium (DMEM, Gibco) supplemented with $10 \%$ (vol/vol) heat inactivated bovine serum (Invitrogen) and $200 \mu \mathrm{g} / \mathrm{ml}$ geneticin (G418 disulfate salt, Sigma). At 70-80\% confluency, the cells were transiently transfected (Effectene, Qiagen) with the A subunit of the human serotonin receptor 5-HT3 (HORF clone collection, Invitrogen) in the pcDNA3.1 $(+)$ vector (Invitrogen) and with the pEGFP-C1 (Clontech, Heidelberg, Germany) plasmid to allow for selection of double-transfected cells in a fluorescence microscope (ratio 1:1 of both plasmids). After $24 \mathrm{~h}$ the cells were split without application of trypsin. The suspension was diluted with DMEM without bovine serum and without geneticin at a ratio of 1:40 to 1:30. After equilibrating the chips with serum-free DMEM for 2 hours at $37^{\circ} \mathrm{C}, 1 \mathrm{ml}$ of the cell suspension was seeded and cultured for 24 hours at $37^{\circ} \mathrm{C}$ in $5 \% \mathrm{CO}_{2}$.

\subsection{Methods}

\subsubsection{Electrophysiology}

For measurements in whole-cell configuration, borosilicate micropipettes were pulled to a resistance of $2-3 \mathrm{M} \Omega$. They were coated with Sylgard (Dow Corning, Midland, MI) and filled with an intracellular electrolyte containing (in $\mathrm{mM}) 140 \mathrm{KCl}, 5 \mathrm{EGTA}$ and $10 \operatorname{HEPES}(p \mathrm{H} 7.3$ adjusted with $\mathrm{KOH}, 290 \mathrm{mOsmol} / \mathrm{lit})$. The extracellular electrolyte contained (in mM) $5.4 \mathrm{KCl}, 135 \mathrm{NaCl}, 1.8 \mathrm{CaCl}_{2}, 1 \mathrm{MgCl}_{2}$,
5 HEPES and 10 glucose $(p \mathrm{H} 7.3$ adjusted with $\mathrm{NaOH}$, $290 \mathrm{mOsmol} / \mathrm{lit}, 62.5 \Omega \mathrm{cm}$ ) (all chemicals from Sigma). We used an Axopatch 200B amplifier (Axoclamp, Union City, $\mathrm{CA}$ ) with an $\mathrm{Ag} / \mathrm{AgCl}$ reference electrode (WPI, Sarasota, FL) in the bath at ground potential. The stray capacitance was completely compensated, the series resistance and membrane capacitance were compensated to $80-90 \%$ with the build-in circuits of the amplifier. The signals were filtered at $10 \mathrm{kHz}$ and sampled at $30 \mathrm{kS} / \mathrm{s}$.

\subsubsection{Transistor recording}

A change of the electrical potential in the extracellular space beneath cultured cells can be detected with an EOSFET [2-5]. The procedure consists of three steps: i) An operating point of the transistor is defined by applying a bias voltage to the bulk silicon and the source with respect to a $\mathrm{Ag} / \mathrm{AgCl}$ electrode on ground potential in the bath. That bias voltage corresponds to the inverted bias of the gate voltage $V_{g s}$. ii) The change of sourcedrain current at the operating point is calibrated in terms of a change of the electrical potential in the electrolyte by modulating the bath potential yielding a transconductance $g_{m}=\Delta I_{D} / \Delta V_{g s}$. iii) Using the transconductance, the change of the source-drain current in a recording experiment is expressed in terms of a change of the electrical potential on the gate and that signal is identified with the extracellular voltage in the cell-chip junction with $V_{J}=\Delta I_{D} / g_{m}$. In our experiments, the bias voltage applied to bulk silicon and source was $+0.5 \mathrm{~V}$ with a gate voltage $V_{g s}=-0.5$. At a voltage of $0.5 \mathrm{~V}$ between source and drain, the source-drain current was around $I_{D}=10 \mu \mathrm{A}$ with a transconductance of about $g_{m}=-20 \mu \mathrm{S}$. In recording experiments, the source-drain current was amplified, filtered at $10 \mathrm{kHz}$ and sampled at $30 \mathrm{kS} / \mathrm{s}$.

\subsubsection{Protocol}

All experiments were performed at room temperature. Individual cells on transistors were selected with a microscope (Axioskop 2 FS; Zeiss, Oberkochen, Germany) that was equipped with a filter set for EGFP fluorescence. The transistor beneath the cell was calibrated. For voltage-clamp and current-clamp experiments, a wholecell pipette contact was established.

The agonist was applied with a double-barrelled glass pipette $(\Theta$ tube) mounted to a solution switching system with a piezo-drive (LSS-3100 Burleigh, Fishers, NY). The $\Theta$ tube was pulled from a two-channel glass tube with $2 \mathrm{~mm}$ outer diameter, $0.3 \mathrm{~mm}$ wall thickness, $0.117 \mathrm{~mm}$ septum (Hilgenberg, Malsfeld, Germany) to a tip diameter of $160-180 \mu \mathrm{m}$. One barrel was filled $100 \mu \mathrm{M}$ serotonin (H-9523, Sigma) in extracellular electrolyte, the other with extracellular electrolyte. The culture chamber was flushed with extracellular electrolyte. In some experiments, the antagonist tropisetron (T-104, Sigma) [17] was added to the extracellular electrolyte at a concentration of 


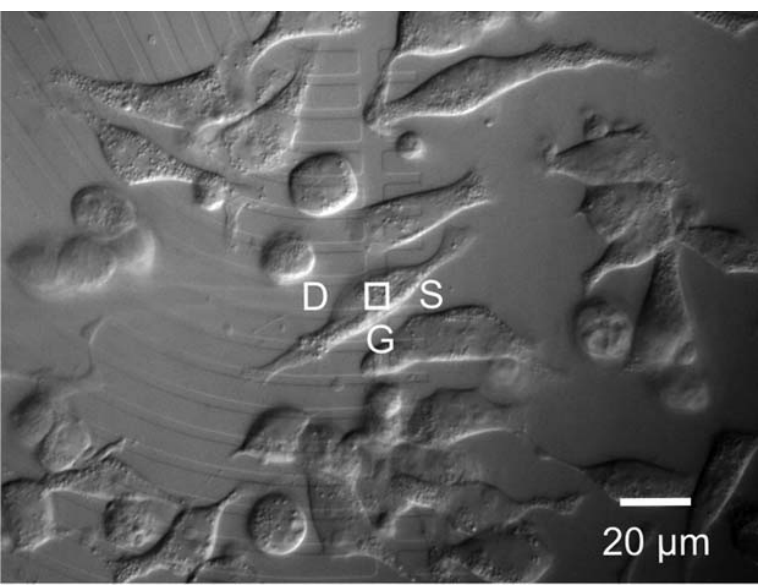

Fig. 3. Receptor-cell-transistor chip. Micrograph (differential interference contrast) of transistor array with HEK293 cells that are transfected with the serotonin receptor 5-HT3A and the potassium channel Kv1.3. For one transistor, the positions of drain (D), gate (G) and source (S) are indicated.

$1 \mu \mathrm{M}$. Between different experiments with the same cell, a period of at least $2 \mathrm{~min}$ allowed for complete washing out of serotonin and for recovery of the receptors.

\section{Results and discussion}

In all experiments, the silicon chips were coated by a layer of adsorbed fibronectin before culturing the cells. Under those conditions, the lipid bilayer of the cells and the oxide of the chip were separated by an extracellular cleft that has a width of about $50 \mathrm{~nm}$ with a resistivity that corresponds to the bath electrolyte [18]. The cleft forms the core of the planar core-coat conductor which transforms currents through the adherent membrane into extracellular voltages on the gate of the transistor. An example of double-transfected HEK293 cells on an array of EOSFETs is depicted in Figure 3.

At first, the interfacing of the serotonin receptor 5-HT3A and of the potassium channel Kv1.3 in doubletransfected HEK293 cells was separately studied under voltage-clamp. Then, under current-clamp the effect of Kv1.3 on the intracellular voltage was investigated when 5 -HT3A is activated by serotonin. Finally, the sensor experiments without patch-pipette were performed, and the results were compared with the voltage-clamp and current-clamp recordings.

\subsection{Voltage-clamp experiments}

The interfacing of 5-HT3A and Kv1.3 in doubletransfected HEK293 cells to EOSFETs was studied at constant intracellular voltage, in analogy to the experiments with cells that were separately transfected with 5-HT3A [5] and Kv1.3 [3].

\subsubsection{Serotonin receptor}

In a first set of experiments, we applied $100 \mu \mathrm{M}$ serotonin at an intracellular voltage of $-70 \mathrm{mV}$ where the Kv1.3 channels are closed. A transient inward current was observed with an amplitude of $I_{M}^{V C}=-3.8 \mathrm{nA}$ (Fig. 4a). Simultaneously, we recorded a transistor signal. The transient voltage had an amplitude of $V_{J}^{V C}=-2.2 \mathrm{mV}$ (Fig. 4a). When we held the intracellular voltage at $V_{M}^{V C}=-120 \mathrm{mV}$, the pipette current as well as the transistor voltage became larger. The waveforms of membrane current and transistor voltage were similar. However, there was a delay of $50 \mathrm{~ms}$ between the maxima as illustrated in the inset of Figure $4 \mathrm{a}$ for the experiment with $V_{M}^{V C}=-120 \mathrm{mV}$.

The membrane current and the transistor signal correspond to the result with HEK293 cells that were transfected only with 5-HT3A [5]. The transient inward current $I_{M}^{V C}$ is typical for the fast activation and slow desensitization of 5-HT3A with a reversal voltage $V_{0}^{\text {rec }}=-5 \mathrm{mV}[19]$. As shown in reference [5], the maximum is determined by the conductance in the free membrane that is activated immediately upon the application of serotonin. Considering equation (1), it is given by

$$
\left(A_{M}-A_{J}\right) g_{F M, \max }^{\mathrm{rec}}\left(V_{M}^{V C}-V_{0}^{\mathrm{rec}}\right)=I_{M, \max }^{V C} .
$$

The adherent membrane is activated with a delay due to the diffusion of serotonin to the area of cell adhesion as discussed in reference [5]. The transient negative voltage on the transistor is dominated by the inward current that flows along the cell-chip junction with a conductance $G_{J}$. Considering equation (2) it is described by

$$
A_{J} g_{J M, \max }^{\mathrm{rec}}\left(V_{M}^{V C}-V_{0}^{\mathrm{rec}}\right)=G_{J} V_{J, \max }^{V C} .
$$

From Figure 4 we obtain a ratio $V_{J, \max }^{V C} / I_{M, \max }^{V C}=$ $0.6 \mathrm{M} \Omega$ of voltage and current. Using equations (9) and (10), a junction conductance $G_{J}=0.83 \mu \mathrm{S}$ is estimated when we assume the same maximum conductance in the free and adherent membrane with $g_{F M, \max }^{\mathrm{rec}}=$ $g_{J M, \max }^{\mathrm{rec}}$ and a hemispherical cell with $A_{J} / A_{M}=1 / 3$. That value is in a typical range for HEK293 cells on chips coated with fibronectin [18].

\subsubsection{Potassium channel}

Without serotonin, we applied depolarizing intracellular voltages (duration $40 \mathrm{~ms}$ ) to the same double-transfected cells that were used for the activation of 5-HT3A, starting at an intracellular voltage of $-70 \mathrm{mV}$. We observed an outward current that slowly decayed (Fig. 4b). Simultaneously, a positive voltage was recorded with the transistor (Fig. 4b). Membrane current and transistor voltage became larger when the depolarization was enhanced. The waveforms of the two kinds of signals were similar. The outward current is typical for a fast activation and slow inactivation of Kv1.3 with a reversal voltage $V_{0}^{\mathrm{K}}=-80 \mathrm{mV}$. With a homogeneous potassium conductance $g_{F M}^{\mathrm{K}}=g_{J M}^{\mathrm{K}}=g_{M}^{\mathrm{K}}$, the maximum current is 
a 5-HT3A

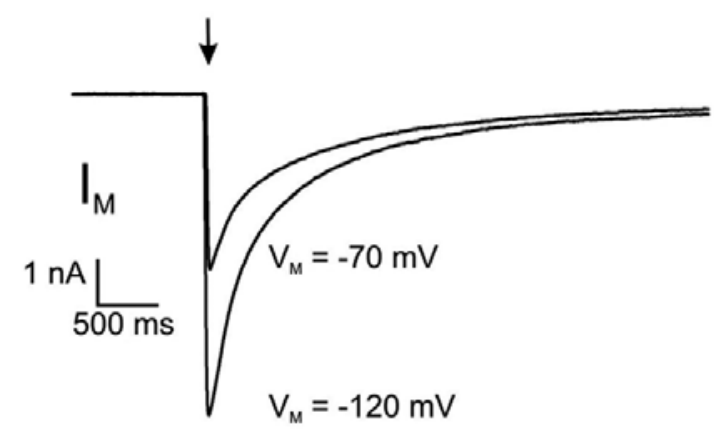

b Kv1.3

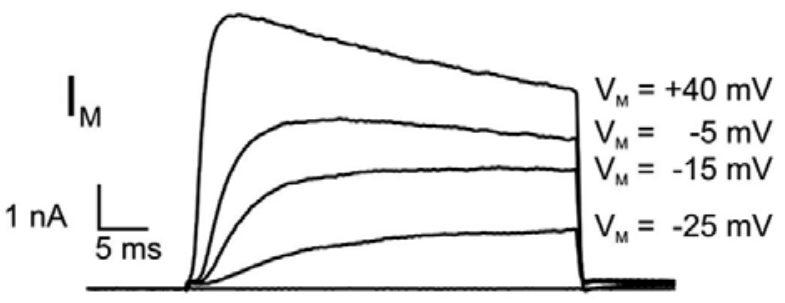

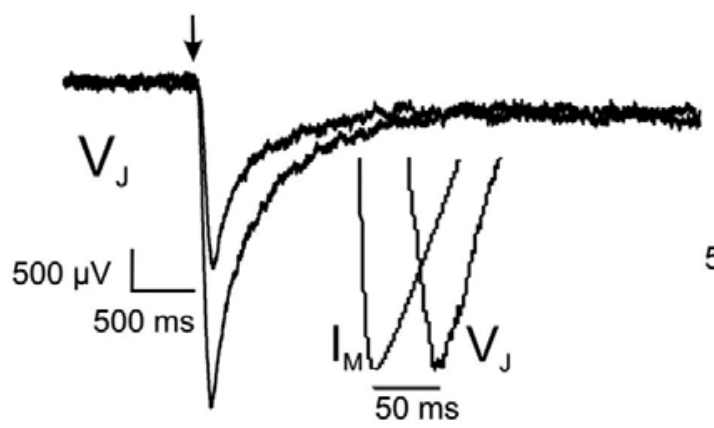

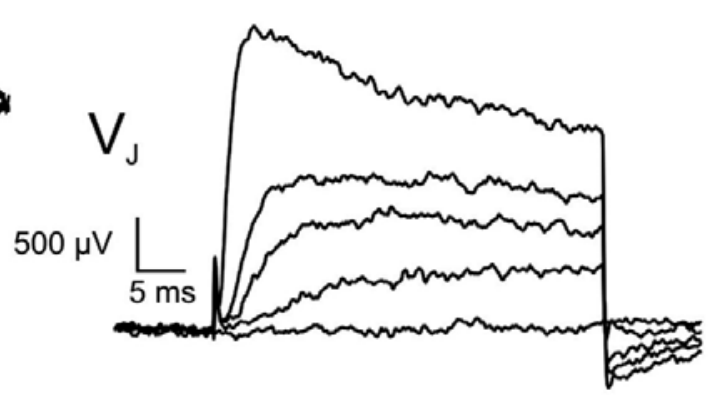

Fig. 4. Voltage-clamp experiment of transistor recording. (a) Activation of 5-HT3A receptor by application of $100 \mu \mathrm{M}$ serotonin (arrow). Recordings of the whole-cell inward current $I_{M}$ (upper traces) and of the extracellular voltage $V_{J}$ (lower traces) for two intracellular voltages $(-70 \mathrm{mV},-120 \mathrm{mV})$. The inset of the lower figure shows the delay between current and voltage at $-120 \mathrm{mV}$ with an expanded time scale. (b) Activation of Kv1.3 channel by depolarizations (duration $40 \mathrm{~ms})$ from $-70 \mathrm{mV}$ to four different voltages. Recordings of whole-cell outward current $I_{M}$ (upper traces) and of the extracellular voltage $V_{J}$ (lower traces).

given by equation (11). The positive voltage on the transistor reflects the current through the adherent membrane according to equation (12).

$$
\begin{aligned}
& A_{M} g_{M, \max }^{\mathrm{K}}\left(V_{M}^{V C}-V_{0}^{\mathrm{K}}\right)=I_{M, \max }^{V C} \\
& A_{J} g_{M, \max }^{\mathrm{K}}\left(V_{M}^{V C}-V_{0}^{\mathrm{K}}\right)=G_{J} V_{J, \max }^{V C}
\end{aligned}
$$

From Figure 4 we obtain a ratio $V_{J, \max }^{V C} / I_{M, \max }^{V C}=$ $0.45 \mathrm{M} \Omega$ of voltage and current. Using equations (11) and (12) for a hemispherical cell, we estimate a junction conductance $G_{J}=0.74 \mu \mathrm{S}$ in the same range as above.

The similarity of the waveforms of membrane current and transistor voltage differs from previous experiments with Kv1.3 [3]. There, a slow component of the transistor signal was observed that was attributed to the binding and unbinding of $\mathrm{K}^{+}$ions to the gate oxide. We observed such a signal also with the double-transfected cells, when the chips were cleaned with the alkaline detergent used in the earlier study. The slow component disappeared if the acidic detergent was used as mentioned in the experimental section. The mechanism of oxide passivation is under investigation. The small negative transistor voltage after stimulation in Figure $4 \mathrm{~b}$ is caused by electrodiffusion of $\mathrm{K}^{+}$ions in the cell-chip junction [3].

\subsection{Current-clamp experiments}

Without patch-pipette, it is the role of the Kv1.3 channels to keep the intracellular voltage below the reversal voltage of the 5-HT3A channels. We checked that effect with a patch-pipette under current-clamp. At first, we applied $100 \mu \mathrm{M}$ serotonin to cells that were transfected only with 5 -HT3A. Starting at $-70 \mathrm{mV}$, the intracellular voltage rapidly approached the reversal voltage $V_{0}^{\text {rec }}=-5 \mathrm{mV}$ of 5-HT3A (Fig. 5). Then we performed the same experiment with double-transfected cells. In that case, the intracellular voltage reached a level of $V_{M}^{C C}=-20 \mathrm{mV}$, distinctly below the reversal voltage (Fig. 5). After electrical relaxation, the intracellular voltage is determined by the balance of ionic inward and outward currents (Eq. (6)).

The current-clamp experiment demonstrates i) that the voltage-gated Kv1.3 channels are opened by the depolarization due to the activation of 5-HT3A receptors, and ii) that the potassium outward current keeps the intracellular voltage below the reversal voltage of the serotonin receptor. After the rapid electrical relaxation, there is a slow repolarization (Fig. 5). It differs for cells without and with Kv1.3. In the first case, it is determined by the desensitization of 5-HT3A, whereas in the second case the desensitization of 5-HT3A and the inactivation of Kv1.3 play a role. 


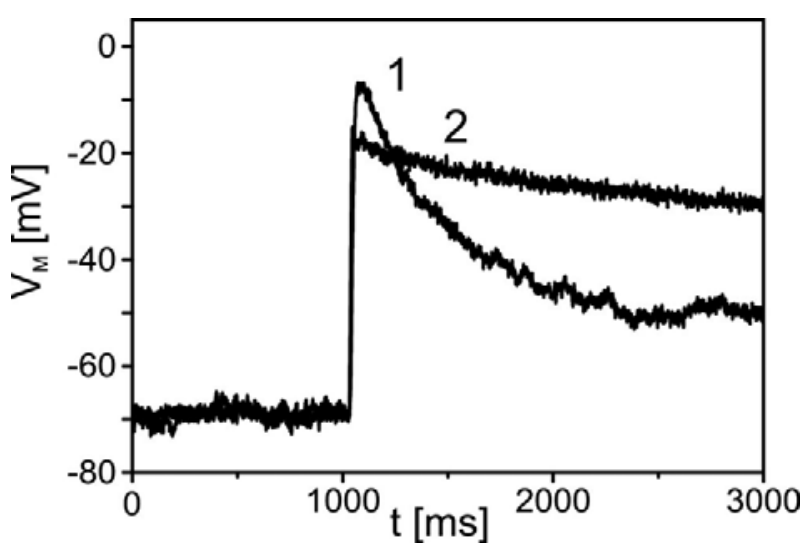

Fig. 5. Current-clamp experiment. Upon the application of serotonin $(100 \mu \mathrm{M}$ at $1000 \mathrm{~ms})$, the intracellular voltage $V_{M}$ is measured with a patch-clamp pipette. 1: HEK293 cell with 5-HT3A receptor without Kv1.3 channel. The rapid depolarization approaches the reversal voltage of $-5 \mathrm{mV}$. 2: HEK293 cell with 5-HT3A receptor and Kv1.3 channel. The depolarization stops below the reversal voltage of 5-HT3A channels. The repolarization in the two systems occurs on a time scale of seconds.

\subsection{Sensor experiments}

The sensor experiments were performed without patchpipette by applying $100 \mu \mathrm{M}$ serotonin to doubletransfected HEK293 cells on transistors.

\subsubsection{Transistor response}

In all experiments, we observed a biphasic transient of the extracellular voltage with a sharp peak and a subsequent wider trough. Four examples of transistor recordings with different cells are shown in Figure 6a. The separation of maximum and minimum was in the order of $100 \mathrm{~ms}$. The signals were almost identical when the stimulation was repeated for the same cell. However, the signals considerably varied for different cells. The integral distribution of the amplitudes is plotted in Figure 6b, separately for the positive and negative signals.

We performed two control experiments: i) After a successful transistor recording upon the application of serotonin with a biphasic response (Fig. 7a), we repeated the stimulation in the presence of tropisetron - a selective antagonist for the serotonin receptor - at a concentration of $1 \mu \mathrm{M}$. Now the transistor signal completely disappeared (Fig. 7b). ii) We used HEK293 cells that were transfected only with 5-HT3A. When we applied serotonin, no transistor response was observed (data not shown). (An experiment with blocked Kv1.3 in double-transfected cells was not possible, because margatoxin and other inhibitors of Kv1.3 surprisingly affected 5-HT3A.) The control experiments prove that the biphasic response of the transistor depends on the simultaneous presence of 5-HT3A and of Kv1.3.
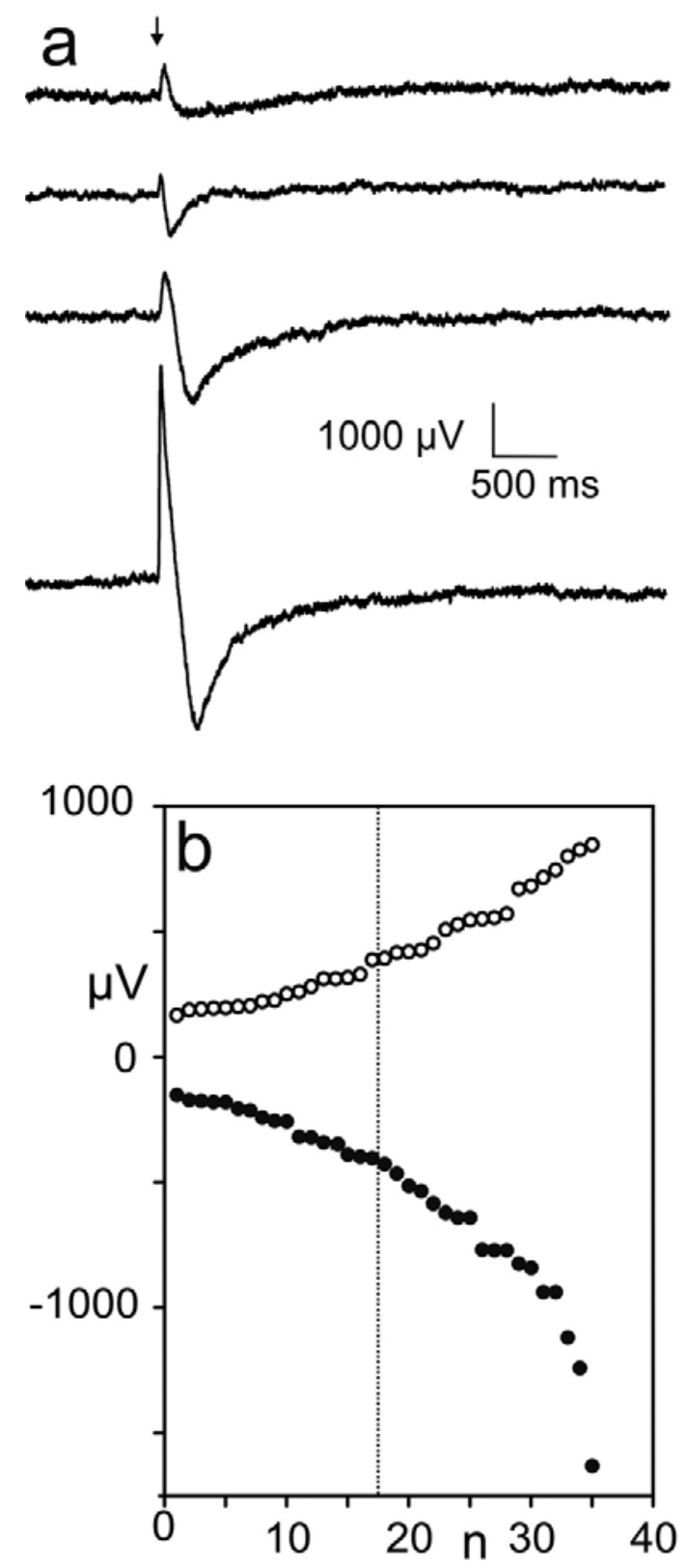

Fig. 6. Sensor experiments without patch-pipette for 5-HT3A receptor and Kv1.3 channel in HEK293 cell. (a) Transistor recordings of four different cells upon the application of $100 \mu \mathrm{m}$ serotonin (arrow). The largest signal belongs to a very large cell. (b) Integral distribution of positive (circles) and negative (dots) amplitudes for 35 cells with medians (dotted line) at $+390 \mu \mathrm{V}$ and $-410 \mu \mathrm{V}$. The exceptionally large signal in (a) is omitted.

\subsubsection{Interpretation}

We assign the extracellular voltage to an ion current that flows through the adherent membrane and along the conductance of the cell-transistor junction, with a positive signal for an outward current, and a negative voltage for an inward current. Considering the voltage-clamp and current-clamp experiments (Figs. 4 and 5), the outward 


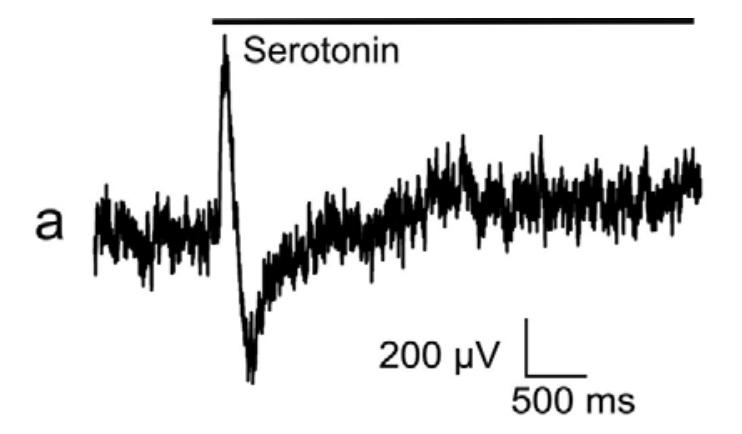

Tropisetron

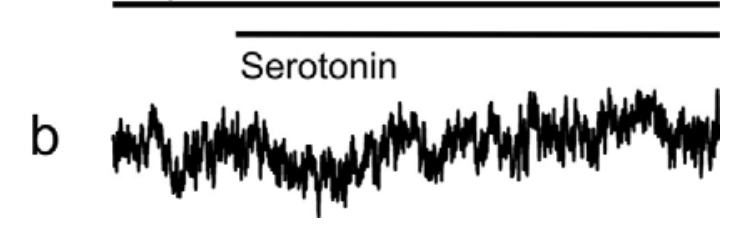

Fig. 7. Selective blocking of 5-HT3A receptor in receptorcell-transistor device. (a) Biphasic transient of transistor signal with 5-HT3A receptor and Kv1.3 channel upon application of $100 \mu \mathrm{M}$ serotonin. (b) Response of the same cell in the presence of $1 \mu \mathrm{M}$ tropisetron, a selective antagonist of the 5 -HT3A receptor.

current results from potassium channels in the adherent membrane whereas the inward current is due to activated 5-HT3A channels in the adherent membrane.

The mechanism is illustrated in Figure 8: I) Immediately after serotonin application, 5-HT3A receptors are activated in the free membrane. An inward current depolarizes the cell such that Kv1.3 channels open in the whole cell. Part of the $\mathrm{K}^{+}$outward current flows through the adherent membrane and gives rise to a positive voltage. II) With a delay due to serotonin diffusion, the 5-HT3A receptors are activated in the adherent membrane while the receptors in the free membrane become desensitized. The inward current through the adherent membrane is partly compensated by a local $\mathrm{K}^{+}$outward current. The remaining net current induces a negative voltage.

The mechanism is described by equation (8) with $V_{M}-V_{0}^{\mathrm{rec}}<0$ in the limits with $g_{J M}^{\mathrm{rec}}=0$ for the first stage and with $g_{F M}^{\mathrm{rec}}=0$ for the second stage. Of course, the two stages are not strictly separated, because the receptors in the adherent membrane become activated before the receptors in the free membrane are completely desensitized. As a consequence, the positive signal is shortened, and the positive and negative amplitudes are lowered by partial compensation.

A crucial test for the consistency of the mechanism is a comparison of the transistor recordings without pipette and the experiments under voltage-clamp and current clamp. Using equations (9) and (2), we obtain the ratio $V_{J, \max } / V_{J, \max }^{V C}$ of the maxima without pipette and under voltage-clamp signal with activated receptor for the two limiting situations. When we substitute the intracellular voltage without pipette by the voltage $V_{M}^{C C}$ under current-

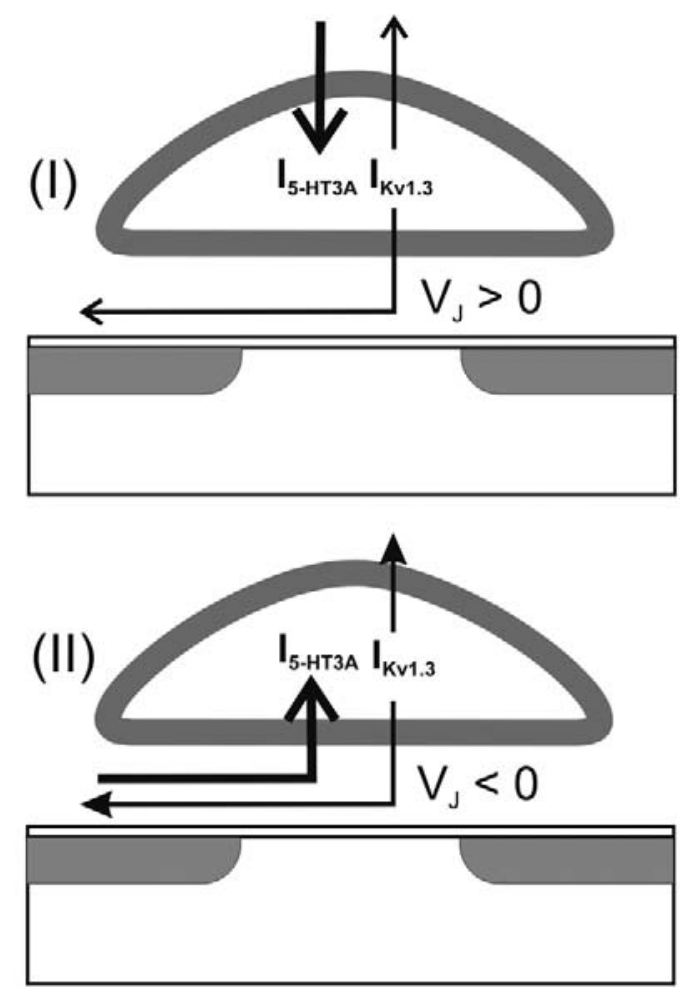

Fig. 8. Mechanism of receptor-cell-transistor device. Stage (I): 5-HT3A receptor channels ared opened in the upper cell membrane. The local inward current drives an outward current through Kv1.3 channels in the whole cell. A voltage $V_{J}>0$ is induced on the transistor. Stage (II): 5 -HT3A channel are opened in the attached membrane. Again an outward current through Kv1.3 channels is induced in the whole cell. A net inward current through the attached membrane gives rise to a voltage $V_{J}<0$.

clamp, we obtain

$$
\mp \frac{V_{M}^{C C}-V_{0}^{\mathrm{rec}}}{V_{M}^{V C}-V_{0}^{\mathrm{rec}}} \frac{A_{M}-A_{J}}{A_{M}}=\frac{V_{J, \max }}{V_{J, \max }^{V C}} .
$$

In the first stage, the sensor signal is opposite to the voltage-clamp signal whereas in the second stage, it has the same sign. The amplitudes of the sensor signal differ from the voltage-clamp signal because different intracellular voltages are induced by activated potassium channels and by a patch-pipette. We insert $V_{M}^{V C}=-70 \mathrm{mV}$ and $V_{J}^{V C}=-2 \mathrm{mV}$ from voltage-clamp experiment of Figure $4 \mathrm{a}$ and $V_{M}^{C C}=-20 \mathrm{mV}$ from the current-clamp experiment of Figure 5 with the reversal voltages $V_{0}^{\text {rec }}=$ $-5 \mathrm{mV}$ and $V_{0}^{\mathrm{K}}=-80 \mathrm{mV}$. For a hemispherical cell with $A_{J} / A_{M}=1 / 3$, we obtain a sensor signal $V_{J} \approx \pm 300 \mu \mathrm{V}$ that is in good agreement with the data in Figure 7.

\subsubsection{Variability}

The wide range of waveforms shown in Figures 6 and 7 is a typical feature of receptor-cell-transistor recording. On the one hand, it is caused by the variability of cell size 
and of the expression of the serotonin receptor and of the potassium channel. On the other hand, it is due to the uncontrolled position of the cells on the transistors. At a given cell size and channel expression, that position affects the voltage on the gate. In a one-compartment model, it modulates the effective conductance $G_{J}$ of the cell-chip junction [4]. With a linear transistor array, the chances to find a cell on a transistor in a suitable position are fairly low, and the number of successful experiments with large signal amplitudes is low. For that reason, we concentrated our study on the implementation of a receptor-celltransistor device and the elucidation of its fundamental mechanism.

To overcome the high variability of the signals in a receptor-cell-transistor device, a large number of unbiased recordings is required that allows a reliable statistical evaluation. This may be achieved by cultivating the cells on densely packed two-dimensional arrays of sensor transistors [6] with a suitable microfluidic application of the agonist. That approach will provide the basis for a more quantitative interpretation of the transient transistor signals and for an evaluation of a dose-response relation that is essential for the development of a practical sensor.

\section{Conclusions}

We have solved the biophysical problem of interfacing ligand-gated ion channels to transistors on the level of individual cells without patch-clamp technique for the ionotropic serotonin receptor 5-HT3A. Crucial were a cotransfection with the potassium channel Kv1.3 that maintained a driving force for the receptor-activated current and an inhomogeneous activation of the 5-HT3A receptors that prevented a local compensation of inward and outward currents. The cell-transistor junction provided a transduction of the ion current to an extracellular voltage on an EOSFET. No signal averaging was required due to a low intrinsic noise of the transistor.

The characterization of the receptor-cell-transistor sensor with the 5-HT3A and Kv1.3 provides a basis for the development of noninvasive sensors in automated high-throughput screening and neurotoxin detection. No involved handling of individual cells and no damage of the cells is required as in patch-clamp systems [20-22]. The approach is not only suitable for other ionotropic receptors, but also for G-protein coupled receptors where an intracellular signal cascade activates the ion channels that are probed by the transistor. For practical applications, the variability of the transistor signals must be overcome by multi-transistor arrays with a reliable statistical evaluation from parallel recordings of a great number of cells.

We thank Marlon Hinner and Ingmar Schoen for critical reading of the early versions of the manuscript. The project was supported by the Federal Minister of Research, and the IST programme of the European Union (NaChip project).

\section{References}

1. P. Fromherz, Solid State Electron. 52, 1364 (2008).

2. B. Straub, E. Meyer, P. Fromherz, Nature Biotech. 19, 121 (2001).

3. M. Brittinger, P. Fromherz, Appl. Phys. A 81, 439 (2005).

4. M. Schmidtner, P. Fromherz, Biophys. J. 90, 183 (2006).

5. I. Peitz, M. Voelker, P. Fromherz, Angew. Chem. Int. Ed. 46, 5787 (2007).

6. A. Lambacher et al., Appl. Phys. A 79, 1607 (2004).

7. A.J. Thomson, S.C.R. Lummis, Curr. Pharm. Des. 12, 3615 (2006).

8. B.J. Jones, T.P. Blackburn, Pharmacol. Biochem. Behav. 71, 555 (2002).

9. P.A. Davies, M. Pistis, M.C. Hanna, J.A. Peters, J.J. Lambert, T.G. Hales, E.F. Kirkness, Nature 397, 359 (1999).

10. F.G. Boess, R. Beroukhim, I.L. Martin, J. Neurochem. 64, 1401 (1995).

11. D.D. Mott et al., J. Physiol. (London) 535, 427 (2001).

12. W. Stuhmer et al., EMBO J. 8, 3235 (1989).

13. S. Marom, I.B. Levitan, Biophys. J. 67, 579 (1994).

14. R. Weis, P. Fromherz, Phys. Rev. E 55, 877 (1997).

15. W.G. Regehr et al., J. Neurosci. Meth. 30, 91 (1989).

16. M. Voelker, P. Fromherz, Small 1, 206 (2005).

17. S. Lankiewicz et al., Mol. Pharmacol. 53, 202 (1998).

18. D. Braun, P. Fromherz, Phys. Rev. Lett. 86, 2905 (2001).

19. M. Barann et al., Arch. Pharmacol. 362, 255 (2000).

20. K.G. Klemic et al., Biosens. Bioelectron. 17, 597 (2002).

21. N. Fertig, R.H. Blick, J.C. Behrends, Biophys. J. 82, 3056 (2002).

22. A. Stett et al., Receptors Channels 9, 59 (2003). 